Europäische Kommission (2007): Employment in Europe 2007, COM(2007) 359 final, Luxemburg

Eurostat Homepage Strukturindikatoren, URL: http://epp.eurostat.ec.europa.eu/portal/page?_ pageid=II33,47800773,II33_47803568\&_dad=portal\&_schema=PORTA

Geisberger, Tamara (2007): Geschlechtsspezifische Lohn- und Gehaltsunterschiede, in: Statistische Nachrichten, Jg. 62, Nr. 7, S. 633-642

Guger, Alois/Aiginger, Karl (2005): Das europäische Gesellschaftsmodell, Wien

Guger, Alois / Marterbauer, Markus (2007): Langfristige Tendenzen der Einkommensverteilung in Österreich - ein Update. Die Verteilung von

Einkommen und Vermögen, WIFO Working Papers, Nr.307/2007

Lehndorff, Steffen (1998): Von der »kollektiven« zur »individuellen« Arbeitszeitverkürzung?, in: WSI Mitteilungen, Jg. 5I, Nr. 9, S. 569-579

OECD (2007): OECD Employment Outlook, Paris: OECD

Plantenga, Janneke/Remery, Chantal (2006): The Gender Pay Gap - Origins and Policy Responses. A Comparative Review of 30 European Countries, Luxemburg: Europäische Kommission

\title{
Arme Schuldner - Reiche Schuldner? Haushaltsverschuldung und Geldvermögen privater Haushalte auf Basis von Mikrodaten \\ Poor Debtors - Rich Debtors? Household Indebtedness and Financial Assets of Private Households Based on Micro Data
}

Pirmin Fessler* und Peter Mooslechner*

\section{Einleitung}

Die Verschuldung privater Haushalte (im Weiteren als Haushalte bezeichnet) hat in den OECD-Ländern langfristig deutlich zugenommen (vgl. Girouard et al. 2006, Barnes und Young 2003). Gleichzeitig stieg jedoch auch das Geldvermögen privater Haushalte markant. Getrennt voneinander haben beide Tendenzen vielfach analytische, aber auch wirtschaftspolitische Aufmerksamkeit gefunden. Simplifiziert wird dabei (medial) immer wieder der Eindruck erweckt, dass Haushalte tendenziell verschuldet sind, wenn (oder weil) sie

* Oesterreichische Nationalbank, Wien. Für wertvolle Kommentare danken die Autoren insbesondere Martin Schürz und Alfred Stiglbauer.

(C) INTERVENTION 5 (I), 2008, 3I-45 
ein niedriges Einkommen und/oder niedriges / kein Vermögen aufweisen. In dieser Arbeit wird auf Basis internationaler Daten der Luxembourg Wealth Study (LWS) sowie einer repräsentativen österreichweiten Haushaltsumfrage der Oesterreichischen Nationalbank (OeNB) 2004 zum Geldvermögen privater Haushalte (vgl. Beer et al. 2006) versucht, dem Zusammenhang zwischen (Geld-)Vermögen und Verschuldung auf Haushaltsebene detaillierter nachzugehen.

Um die Belastung von Haushalten durch ihre Verschuldung - und die damit verbundenen Implikationen für Finanzmarktstabilität - genauer analysieren zu können, ist es wichtig, die Verschuldungssituation des Haushalts und sein Geldvermögen gemeinsam zu analysieren. Makroökonomische Daten erlauben es lediglich, die Expansion der Verschuldung und gleichzeitig des Geldvermögens des Haushaltssektors nachzuvollziehen. Makroökonomische Daten liefern jedoch keine Rückschlüsse darauf, welche Haushalte sich verschulden und welche Geldvermögen akkumulieren (vgl. Cox et al. 2002). Diese Informationen sind jedoch wesentlich, wenn Unterschiede in der Belastung der Haushalte durch Verschuldung analysiert werden sollen. Halten vor allem solche Haushalte Schulden, die fehlendes Einkommen oder Vermögen substituieren, so ist dies für die Finanzmarktstabilität besonders relevant. Ist Verschuldung hingegen vor allem ein Merkmal von Haushalten mit hohem Einkommen und hohem Vermögen, ist die Situation als weit weniger problematisch einzuschätzen, da diese Haushalte "Finanzschocks" - z. B. höhere Kreditzinssätze - besser abfedern können.

Cox et al. (2002) stellen für das Vereinigte Königreich fest, dass das Verhältnis von Schulden zu Vermögen mit steigendem Vermögen sinkt. Absolut sind aber gerade jene Haushalte am meisten verschuldet, die auch das höchste Vermögen aufweisen. Zu ähnlichen Ergebnissen kommen Aizcorbe et al. (2003) für die USA: Sowohl der Anteil der verschuldeten Haushalte als auch die Höhe der Verschuldung steigen mit dem Einkommen und dem Vermögen der Haushalte. Beer/Schürz (2007) bestätigen diese Ergebnisse auch für Österreich: mit steigendem Einkommen steigt die Wahrscheinlichkeit, dass ein Haushalt Schulden aufweist. Hinsichtlich des Vermögens sind die Ergebnisse für Österreich, wenn zwischen Wohn- und Konsumkrediten unterschieden wird, nicht eindeutig.

Das gleichzeitige Halten von Schulden und Geldvermögen (in beträchtlichem Ausmaß) ist mikroökonomisch nicht leicht zu erklären. So ist beispielsweise für Verschuldung im Normalfall von höheren Zinssätzen auszugehen als für das Halten von Vermögenswerten. Die möglichen Erklärungsansätze innerhalb traditioneller ökonomischer Ansätze dazu sind limitiert. Am einfachsten erscheint die Erklärung dann, wenn die Zinsen für Verschuldung niedriger sind als die Rendite auf das veranlagte Vermögen. ${ }^{1}$ Eine andere Möglichkeit besteht darin, dass die Haushalte ein gewisses Ausmaß an Liquidität benötigen, die mit dem Halten von Vermögen bei gleichzeitiger Verschuldung einhergeht. Ebenso könnte Geldvermögen z. B. aus Renditegründen - so illiquide gehalten werden, dass es zur Tilgung von Schulden nicht unmittelbar herangezogen werden kann. Insgesamt bleibt jedoch der Erklärungsgehalt dieser Ansätze gering.

I Davon ist zwar im Normalfall nicht auszugehen, spezielle Marktgegebenheiten - etwa im Fall von Fremdwährungskrediten - können jedoch durchaus eine derartige Situation herbeiführen. 
Neben weiteren Erklärungsansätzen aus der Literatur zum Sparen der Haushalte und der Behavioral Economics-Literatur (vgl. Browning/Lusardi 1996, Thaler 1990) stellt das ebenfalls aus der Behavioral Economics-Literatur stammende Konzept des Hyperbolic Discounting (vgl. Laibson et al. 2000, Laibson 1997) einen neueren theoretischen Versuch konkret bezogen auf das gleichzeitige Halten von Kreditkartenschulden und Vermögen in den USA dar. Dieses geht vor allem davon aus, dass für ein Individuum der Diskontsatz für die nähere Zukunft höher ist als der für die längere Frist. Aus den daraus folgenden dynamisch inkonsistenten Präferenzen lässt sich das gleichzeitige Halten von Schulden und illiquiden Vermögenspositionen teilweise erklären. Crook (2003) bietet beispielsweise einen Überblick über Studien zum Angebot an und zur Nachfrage nach Haushaltsverschuldung unter diesen Gesichtspunkten.

Die vorliegende Arbeit gibt in Abschnitt 2 einen Überblick über die logischen Zusammenhänge zwischen Vermögen und Verschuldung auf makroökonomischer Ebene. Anschließend werden in Abschnitt 3 die sozioökonomischen Charakteristika der Haushalte mit Wohn- bzw. Konsumkreditverschuldung sowie der Zusammenhang zwischen Verschuldung und Geldvermögen auf Mikroebene detaillierter betrachtet. Abschließend werden in Abschnitt 4 einige vorläufige Schlussfolgerungen gezogen.

\section{Die Makroebene: Logik des parallelen Wachstums von Finanzaktiva und Finanzpassiva}

In makroökonomischer Perspektive werden Haushaltsverschuldung und das Geldvermögen der privaten Haushalte überwiegend anhand von voneinander unabhängigen Fragestellungen analysiert. Stellt die Haushaltsverschuldung beispielsweise einen regelmäßigen Bestandteil der Analyse des privaten Konsums und der Wohnbauinvestitionen dar, so fließen Überlegungen zur Entwicklung und zur Rolle des privaten Geldvermögens höchstens indirekt in die konjunkturelle Analyse ein. Etwas anders stellt sich die Situation in der Finanzmarktstabilitätsanalyse dar, die gleichzeitig Risiken der Verschuldungsentwicklung und etwa Risiken aus der Veranlagungsstruktur des Geldvermögens der privaten Haushalte zu berücksichtigen hat.

Vor diesem Hintergrund zeigen die Makrodaten der Gesamtwirtschaftlichen Finanzierungsrechnung für nahezu alle Länder und Untersuchungszeiträume einen parallelen tendenziellen Anstieg von Geldvermögen und Verschuldung des Haushaltssektors. Das widerspricht weitgehend der vor allem medial verbreiteten Darstellung, die Haushaltsverschuldung einerseits als primär negativ und andererseits als Konsequenz mangelnden Einkommens und mangelnden Geldvermögens interpretiert. Die grundsätzliche ökonomische Logik von Finanzmarkttransaktionen bzw. von Forderungen und Verpflichtungen im gesamtwirtschaftlichen Zusammenhang macht jedoch unmittelbar deutlich, dass steigendes Geldvermögen und steigende Verschuldung keinesfalls ein Rätsel der ökonomischen Entwicklung darstellen, sondern dass sie die logische Konsequenz wachsender Finanzmärkte sind.

Alle Finanzmarkttransaktionen sind dadurch gekennzeichnet, dass es sich um "zweiseitige "Transaktionen handelt, durch die jeweils gleichzeitig eine Forderung und eine Verbindlichkeit - in gleicher Höhe - entsteht. Gibt - im einfachsten Fall - A einen Kredit in Höhe von Ioo an B, so verfügt A dann über eine Forderung an B in Höhe von Ioo und B 
hat eine Verbindlichkeit gegenüber A ebenfalls in Höhe von Ioo. Für A stellt diese Forderung sein Geldvermögen dar, für B seine Verschuldung. Die Existenz von Finanzintermediären (wie Banken, Versicherungen oder Investmentfonds) in modernen Volkswirtschaften ändert nichts an diesem grundlegenden Prinzip, es tritt lediglich ein Intermediär zwischen den (originären) Gläubiger und den (originären) Schuldner. Im Fall einer Bank tätigt A z. B. eine Einlage bei der Bank in Höhe von Ioo und die Bank gibt diese Einlage in Form eines Kredits an B weiter. Insgesamt bewirkt somit der logisch zweiseitige Charakter von Finanztransaktionen das parallele Wachstum von Finanzaktiva (=Geldvermögen) und Finanzpassiva (=Verschuldung). Auf sektoraler Ebene sind Unterschiede im Wachstum von Geldvermögen und Verschuldung sehr wohl möglich. So ist der Haushaltssektor strukturell durch einen substantiellen Überschuss an Geldvermögen gekennzeichnet, der seinerseits zur Finanzierung der Investitionstätigkeit des Unternehmenssektors dient, der deshalb einen entsprechenden Verschuldungsüberhang aufweist. Parallel zum wachsenden Einkommen steigen aber - im Normalfall - das Geldvermögen und die Verschuldung sowohl im Haushaltswie im Unternehmenssektor, da höhere Einkommen und eine positive Sparquote wachsende Finanztransaktionen ermöglichen.

Für die Gesamtwirtschaft - vereinfacht hier für eine geschlossene Volkswirtschaft gilt folglich:

(I) Summe GV = Summe VS.

Sektoral setzen sich sowohl das Geldvermögen (GV) als auch die Gesamtverschuldung (VS) einer Volkswirtschaft aus den Beiträgen der einzelnen Sektoren, wie Haushalte $(\mathrm{HH})$, Unternehmen (UN) und Staat (ST), zusammen:

(2) Summe GV = GVHH + GVUN + GVST,

(3) Summe VS = VSHH + VSUN + VSST,

wobei die sektoralen Salden, z. B. GVHH - VSHH, ungleich Null sind bzw. sein können.

Daraus ergibt sich aus makroökonomischer Perspektive die Logik einer Parallelität des Wachstums von Geldvermögen und Verschuldung, deren eigentlich relevante und tiefgehende Analyse aber erst auf der Grundlage von Mikrodaten zur finanziellen Situation einzelner Haushalte möglich ist.

\section{Die Mikroebene: Wechselseitige Abhängigkeit von Haushaltsverschuldung und Geldvermögen}

Ein Aspekt der Lebenszyklushypothese des Konsum- und Sparverhaltens (vgl. Modigliani / Brumberg 1954) ist, dass das Alter einen wichtigen Faktor für die Nachfrage nach Krediten und Haushaltsverschuldung darstellt. Junge Haushalte mit einem hohen Grenznutzen des Konsums und mit der Erwartung eines steigenden Einkommens weisen eine hohe Nachfrage nach Krediten auf, die dann mit höherem Alter wieder abnimmt.

Das im Haushalt vorhandene Geldvermögen sollte ebenfalls einen wichtigen Faktor für die Nachfrage nach Verschuldung darstellen. Bei hohem bzw. steigendem Geldvermögen steht dem Haushalt hohe potentielle Kaufkraft zur Verfügung, was generell die Notwen- 
digkeit von Kreditaufnahme und Verschuldung verringert. Je liquider das Geldvermögen gehalten wird, desto stärker sollte dieser Effekt sein. Dem gegenüber steht die Tatsache, dass ein bestimmtes Niveau an Geldvermögen die Nachfrage nach Krediten bzw. Verschuldung erst ermöglicht. Für den Kauf einer Eigentumswohnung ist beispielsweise Voraussetzung, dass ein bestimmtes Ausmaß an Eigenkapital aufgebracht werden kann, bevor für den Rest des Kaufpreises eine Kreditfinanzierung zugebilligt werden wird. Geldvermögen kann ebenso zur Besicherung von Schulden dienen und in diesem Sinne eine vorhandene Kreditbeschränkung überwinden.

Das in der Bevölkerung primär verbreitete Verständnis von Haushaltsverschuldung setzt in erster Linie an der nachfrageseitigen Perspektive an. Kreditaufnahme und Verschuldung werden dabei substitutiv zu Einkommen und Vermögen des Haushalts gesehen, mangelndes Einkommen und mangelndes Vermögen bedingen eine kompensatorische Kreditnachfrage, die die mangelnde Kaufkraft des Haushalts ausgleicht. Diese Sicht vernachlässigt jedoch die angebotsseitigen Gesichtspunkte des Kreditmarktes. Kreditnehmer werden sich nur dann tatsächlich verschulden können, wenn auch die Anbieter von Kredit bereit sind, den Kreditnehmer zu akzeptieren und die entsprechende Verschuldung bereitzustellen. Dabei ist entscheidend, dass sowohl die Einkommenssituation als auch die Vermögenssituation des Haushalts wesentliche Kriterien in der Kreditvergabeentscheidung des Kreditgebers darstellen.

Wie die vorhandenen Daten zeigen, verschulden sich die meisten Haushalte besonders dann, wenn sie Wohneigentum erwerben. Das Volumen von Wohnkrediten ist im Allgemeinen deutlich höher als das von Konsumkrediten. Die Schaffung von Wohnraum und die damit zusammenhängende Verschuldung erfolgt in den meisten Fällen im mittleren Alter des Haushalts(vorstandes). Liquiditätsüberlegungen bedingen gleichzeitig, dass ein Haushalt parallel zur Verschuldung in einem gewissen Ausmaß Geldvermögen hält. Trotzdem ist grundsätzlich bei den Haushalten mit Verschuldung ein negativer Zusammenhang zwischen Geldvermögen und Verschuldung zu erwarten - Verschuldung wird langsam abgebaut und Geldvermögen langsam (wieder) aufgebaut.

Ein wesentlicher Gesichtspunkt in diesem Zusammenhang sind Einkommenserwartungen, die eine Form der »dynamischen Besicherung « von Verschuldung darstellen. Je steiler die Erwartung des zukünftigen Einkommens, umso höher ist zu einem gegebenen Zeitpunkt die Kreditnachfrage. Ist die Einkommenserwartung hingegen eher flach, dämpft das gleichermaßen die angebots- wie die nachfrageseitigen Faktoren der Verschuldung. Gleichzeitig ist mit dem Einkommen auch ein entscheidender Unsicherheitsaspekt verbunden. Steigt die Wahrscheinlichkeit, arbeitslos zu werden, werden Angebot an und Nachfrage nach Krediten sinken. Auch unter Einkommensgesichtspunkten ist es damit insgesamt nicht eindeutig, wie sich das momentane Einkommen des Haushalts auf seine künftige Verschuldungssituation auswirkt. Einerseits könnte ein niedriges Einkommen junger Haushalte mit einem starken zukünftigen Anstieg verbunden sein, andererseits ist der Grad an Unsicherheit in niedrigen Einkommensstufen meist deutlich höher als in höheren Einkommensstufen. Grundsätzlich ermöglicht aber ein höheres Einkommen ein höheres Ausmaß an Haushaltsverschuldung. 
Eine Reihe von Faktoren werden als wichtige strukturelle Indikatoren für die zukünftige Verschuldungsfähigkeit eines Haushalts verwendet: Als Indikator für den zukünftigen Verlauf des Einkommens spielt Bildung eine wichtige Rolle. Je höher das Bildungsniveau des Haushalts, umso eher ist mit einem zukünftigen Anstieg des Einkommens zu rechnen. In Regionen mit hoher Arbeitslosigkeit sollte die Nachfrage nach Krediten geringer sein, gleichzeitig sind Kreditbeschränkungen aufgrund von geringem Einkommen oder Vermögen wichtig. Speziell bei niedrigen Einkommen und Vermögen sollten diese Kriterien wichtig für Kreditaufnahme und deren Höhe sein, da Kreditinstitute potentielle Kreditnehmer nach entsprechenden Richtlinien evaluieren. Wichtig ist wiederum, dass dieselben Variablen sowohl die Nachfrage als auch das Angebot beeinflussen.

Allerdings weisen die großen Unterschiede im Anteil verschuldeter Haushalte und in der Höhe der Haushaltsverschuldung in verschiedenen Ländern darauf hin, dass auch nicht direkt beobachtbare Variablen - wie etwa historische, institutionelle und gesellschaftliche Gründe, die die Einstellung in Bezug auf Wohneigentum und Schulden im Allgemeinen beeinflussen - eine wichtige Rolle spielen.

Ebenso relevant, insbesondere wenn unterschiedliche Länder verglichen werden, könnten gesetzliche Rahmenbedingungen wie steuerliche Absetzmöglichkeiten oder Förderungen (Wohnbauförderungen, Mietzuschüsse, usw.) sein.

\section{Wohnkredite sind bestimmend für die Haushaltsverschuldung im Lebenszyklus}

Eine repräsentative österreichweite Haushaltsumfrage ${ }^{2}$ der OeNB zum Geldvermögen privater Haushalte ermöglicht die Identifizierung von Haushalten, die Wohn- bzw. Konsumkredite halten. ${ }^{3}$ Die sozioökonomischen Charakteristika beziehen sich teilweise auf den Haushalt (z. B. Anzahl der im Haushalt lebenden Personen), teilweise auf die als "Haushaltsvorstand « ${ }^{4}$ identifizierte Person (z. B. Alter). Unterstrichen werden muss, dass Querschnittsdaten grundsätzlich keine Rückschlüsse auf das Verhalten der Haushalte im Lebenszyklus zulassen. Sie können jedoch Hinweise auf die Verschuldungssituation von Haushalten unterschiedlicher Kohorten in relativ ähnlichen Lebenssituationen liefern.

29 Prozent der österreichischen Haushalte besitzen einen Wohnkredit. Der durchschnittliche Wohnkredit beträgt 57.708€ (Median: 36.000€). Wohnkredite sind besonders stark in der Altersgruppe von 30 bis 39 Jahren vertreten, zudem halten Bezieher höherer Einkommen tendenziell eher einen Wohnkredit. Nach Bruttogeldvermögensdezi-

2 Die Stichprobe umfasst 2.556 Haushalte. In dieser Arbeit werden jeweils die für Österreich gewichteten Daten verwendet.

3 Wohnkredite sind Kredite, die zur Beschaffung und Erhaltung von Wohnraum aufgenommen werden (Finanzierung von Häusern bzw. Wohnung sowie An- oder Umbauten derselben). Konsumkredite sind alle anderen Kredite (etwa auch zur Einrichtung einer Wohnung bzw. eines Hauses). 4 In der OeNB Befragung 2004 ist jenes Haushaltsmitglied - geschlechtsunabhängig - als "Haushaltsvorstand" definiert, das entweder als solches von der befragten Person identifiziert wird oder jenes, das »über die finanziellen Angelegenheiten des Haushaltes am besten Bescheid weiß«. 
len $^{5}$ halten zwar tendenziell in niedrigeren Dezilen weniger Haushalte einen Wohnkredit, in den obersten Dezilen sinkt der Anteil jedoch - liegt aber erst im zehnten Dezil wieder unter dem Durchschnitt (vgl. Tabelle I). Der Anteil der Haushalte, die innerhalb einer bestimmten Bruttogeldvermögensklasse einen Wohnkredit halten, lässt nicht auf einen positiven Zusammenhang zwischen Einkommen bzw. Vermögen und Wohnkrediten schließen. ${ }^{6}$ Allerdings steigt mit höherem Bruttogeldvermögen (BGV) die Höhe der Wohnkreditverschuldung an, was einen positiven Zusammenhang bedeutet. Es verschulden sich also auch Haushalte für Wohnzwecke, die eigentlich über ausreichend Bruttogeldvermögen verfügen. Während im ersten BGV-Dezil die Höhe der Wohnkreditverschuldung jene des Bruttogeldvermögens noch circa um das I8-fache übersteigt, liegt das Verhältnis im Io. Dezil bei durchschnittlich nur mehr 20 Prozent. Die Belastung für Haushalte in den unteren Dezilen ist demnach deutlich höher, wobei Alterseffekte im Sinne der Lebenszyklushypothese eine große Rolle spielen.

\section{Konsumkredite sind vorrangig im Bereich der niedrigen Geldvermögen relevant}

Etwa I7 Prozent der österreichischen Haushalte halten Konsumkredite. Die Höhe des durchschnittlichen Konsumkredits liegt bei circa I6.845€ (Median: Io.00o€) und ist damit deutlich niedriger als ein durchschnittlicher Wohnkredit. Tendenziell sind Konsumkreditnehmer jünger als Wohnkreditnehmer. Auch für die Konsumkreditverschuldung gilt, dass sie eher unter den besser verdienenden Haushalten anzutreffen ist. Nach BGV-Dezilen gibt es aber deutliche Unterschiede zu den Wohnkrediten: Konsumkreditnehmer finden sich überdurchschnittlich oft in niedrigen BGV-Dezilen und sind in den oberen BGV-Dezilen tendenziell unterdurchschnittlich vertreten (vgl. Tabelle I, S. 38). Selbst im obersten Dezil hält jedoch noch mehr als jeder ıo. Haushalt einen Konsumkredit. Die Höhe des durchschnittlichen Konsumkredits steigt mit dem BGV, jedoch nicht so ausgeprägt wie bei den Wohnkrediten. Im obersten Bereich der BGV-Verteilung spielen Konsumkredite kaum mehr eine Rolle. Ebenso fällt auf, dass das niedrigste Dezil des Nettogeldvermögens (NGV = BGV abzüglich Kredite) ein höheres durchschnittliches BGV aufweist als das zweite NGV-Dezil. Ursache dafür ist, dass Haushalte am unteren Ende der BGV-Verteilung nur in geringem Ausmaß Wohnkredite halten. Dafür dürften sowohl Alterseffekte als auch Kreditbeschrän-

5 Das Bruttogeldvermögen (BGV) ist definiert als die Summe der Beträge auf Girokonten, Spareinlagen inklusive Bausparverträgen, Wert der Anleihen, Wert der Aktien, Wert der Investmentzertifikate, Wert der Unternehmensbeteiligungen und der gesamten Prämieneinzahlungen in Lebensversicherungen. Alle in dieser Arbeit präsentierten Ergebnisse in Bezug auf das BGV bleiben auch mit einer Definition desselben ohne die Miteinbeziehung der Lebensversicherungen nahezu unverändert. Die Bruttogeldvermögensdezile wurden über die gesamte Stichprobe erstellt und nicht nur jeweils für Wohn- bzw. Konsumkreditnehmer.

6 Eine wichtige Rolle spielt in diesem Zusammenhang, dass in der Erhebung nur Geldvermögen erfasst wurde, während etwa für die Besicherung von Wohnkreditverschuldung Immobilienvermögen wesentlich ist. 


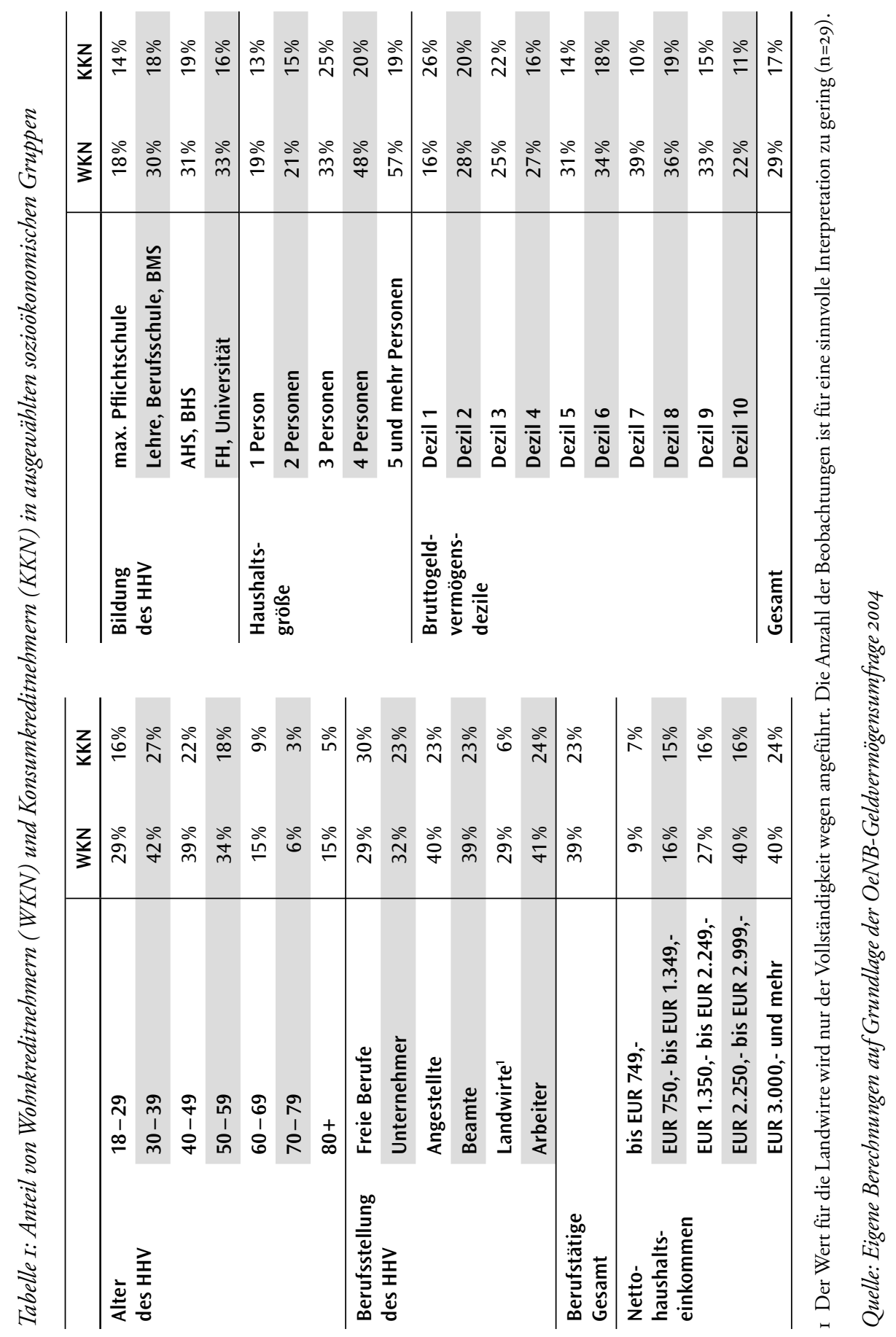


kungen verantwortlich sein, da diesen Haushalten entsprechende Eigenmittel fehlen, um etwa einen Kredit zum Kauf einer Wohnung oder eines Hauses überhaupt aufnehmen zu können. Leider lassen die österreichischen Daten aber eine gleichzeitige Modellierung der Angebotsseite nicht zu, wie sie etwa Magri (2002) für Italien liefert.

\section{Empirische Evidenz zum Zusammenhang von Haushaltsverschuldung und Geldvermögen für Österreich und ausgewählte OECD Länder}

Der Anteil der verschuldeten Haushalte ist in ausgewählten Ländern markant unterschiedlich. In Österreich besitzen etwa 4I Prozent aller Haushalte entweder Wohn- und/oder Konsumkredite. Im internationalen Vergleich stellt das (laut LWS) nur einen unterdurchschnittlichen Wert dar. Lediglich in Deutschland liegt dieser Anteil mit ca. 32 Prozent niedriger, in allen anderen Vergleichsländern hingegen deutlich höher (Finnland ca. 52 Prozent, Vereinigtes Königreich ca. 57 Prozent, USA ca. 68 Prozent und Schweden ca. 70 Prozent). Insbesondere in Schweden und den USA liegt der Anteil von Haushalten mit Krediten besonders hoch. Die Situation in Deutschland ist vermutlich mit der in Österreich am ehesten vergleichbar, allerdings ist der Wert für Deutschland nach unten verzerrt, da die Daten in Deutschland nur ab einer unteren Grenze von $2.500 €$ erhoben werden.

Tabelle 2 (S.40) zeigt die Korrelationen zwischen der Verschuldung der Haushalte mit dem Bruttogeldvermögen bzw. dem verfügbaren Einkommen. ${ }^{7}$ Die Korrelation der Verschuldung mit dem Einkommen ist durchwegs stärker ausgeprägt als mit dem Bruttogeldvermögen. Für die Haushalte mit Verschuldung sind diese Korrelationen für alle ausgewählten Länder positiv und signifikant. Für die verschuldeten Haushalte gilt also, dass tendenziell Haushalte mit höherem Einkommen und höherem Bruttogeldvermögen auch mehr verschuldet sind. Bezieht man in die Berechnungen auch jene Haushalte ein, die keine Verschuldung ausweisen, so bleibt das Einkommen positiv und signifikant für alle Länder mit dem Schuldenstand korreliert, für Deutschland, das Vereinigte Königreich und die USA trifft das auch für das Geldvermögen zu. Für Finnland ist das Geldvermögen dann interessanterweise signifikant negativ mit dem Schuldenstand korreliert, für Österreich und Schweden wird die Korrelation insignifikant.

7 Es handelt sich um die logarithmierte Verschuldung jener Haushalte, die Schulden halten, sowie um das logarithmierte verfügbare Einkommen und das logarithmierte BGV, wobei bei Einkommen und BGV Null-Werte in den logarithmierten Variablen wiederum auf Null gesetzt wurden. Analog vorgegangen wurde in Bezug auf die Verschuldung auch für die zweite Spalte der Tabelle 2. Zudem wird in Tabelle 2 für das BGV die LWS-Variable Total Financial Assets (TFAI) verwendet, was bedeutet, dass das BGV auch für Österreich keine Lebensversicherungen enthält, da diese Daten nicht für alle Länder vorhanden sind. Als Einkommensvariable wurde die LWS-Variable LIS Disposable Income (LIS_DPI) verwendet. Für Österreich existiert zudem keine vergleichbare Einkommensvariable. Alternativ kann auch der Spearman Rangkorrelationskoeffizient für die nicht-logarithmierten Daten verwendet werden. Die Korrelationen bleiben dabei (in Bezug auf die verschuldeten Haushalte) für alle Länder positiv und signifikant. 
Tabelle 2: Korrelationen von Haushaltsverschuldung mit Einkommen und Geldvermögen

\begin{tabular}{|c|c|c|}
\hline & Schuldenstand & $\begin{array}{c}\text { Schuldenstand } \\
(\text { inkl. keine Verschuldung }=0)\end{array}$ \\
\hline \multicolumn{3}{|l|}{ Deutschland } \\
\hline Bruttogeldvermögen & $0.20 * * *$ & $0.03 * * *$ \\
\hline verfügbares Einkommen & $0.44 * * *$ & $0.40 * * *$ \\
\hline \multicolumn{3}{|l|}{ Finnland } \\
\hline Bruttogeldvermögen & $0.13 * * *$ & $-0.07 * * *$ \\
\hline verfügbares Einkommen & $0.36 * * *$ & $0.29 * * *$ \\
\hline \multicolumn{3}{|l|}{ Österreich } \\
\hline Bruttogeldvermögen & $0.30 * * *$ & -0.02 \\
\hline \multicolumn{3}{|l|}{ Schweden } \\
\hline Bruttogeldvermögen & $0.25 * * *$ & 0.00 \\
\hline verfügbares Einkommen & $0.40 * * *$ & $0.45^{* * *}$ \\
\hline \multicolumn{3}{|l|}{ Vereinigtes Königreich } \\
\hline Bruttogeldvermögen & $0.28 * * *$ & $0.09 * * *$ \\
\hline verfügbares Einkommen & $0.35^{* * *}$ & $0.37 * * *$ \\
\hline \multicolumn{3}{|l|}{ USA } \\
\hline Bruttogeldvermögen & $0.29 * * *$ & $0.26 * * *$ \\
\hline verfügbares Einkommen & $0.47 * * *$ & $0.42 * * *$ \\
\hline
\end{tabular}

${ }^{* * *}$ signifikant auf I\%igem Signifikanzniveau

Quelle: Eigene Berechnungen auf Grundlage von LWS (Luxembourg Wealth Study) Daten

Logit-Schätzungen von Beer und Schürz (2007) haben gezeigt, dass Geldvermögen sich signifikant negativ auf die Wahrscheinlichkeit Konsumkredite zu halten und - je nach Geldvermögensklasse unterschiedlich - auf die Wahrscheinlichkeit Wohnkredite zu halten auswirkt.

Die Lebenszyklushypothese legt nahe, dass sich junge Haushalte verschulden und diese Verschuldung dann mit zunehmendem Alter (und Einkommen) wieder abbauen. Gleichzeitig wäre zu erwarten, dass das Einkommen und das Haushaltsvermögen bis zum Ausscheiden aus dem Arbeitsmarkt ansteigen. Aber auch unabhängig von der Lebenszyklushypothese kann argumentiert werden, dass Haushalte, die einen Kredit aufnehmen, ab diesem Zeitpunkt aus Effizienzüberlegungen kein oder sehr wenig Geldvermögen halten werden, bis die Verschuldung in einem wesentlichen Ausmaß getilgt ist. Zu erwarten wäre demnach eine negative Korrelation von Schulden und Geldvermögen zumindest für jene Haushalte, die Schulden halten. 
Nahe liegend als eine Erklärung für die positive Korrelation von Schulden und Geldvermögen ist die fehlende Verschuldungsfähigkeit von Haushalten mit besonders niedrigen Vermögen. Dabei handelt es sich aber um einen zu kleinen Anteil, als dass dieser die positive Korrelation für die Gesamtheit der Haushalte befriedigend erklären könnte. Möglicherweise sind jedoch andere Variablen als das Geldvermögen für die positive Korrelation verantwortlich, selbst dann stellen die vorliegenden Ergebnisse aber einen Hinweis auf suboptimale Allokationen in den Portfolios der Haushalte dar. Gleichzeitig entschärft die Tatsache, dass nicht jene Haushalte hohe Beträge in Schulden halten, die über wenig Geldvermögen verfügen, die Problematik der steigenden Haushaltsverschuldung auf Makroebene.

Ein Vergleich der Verteilungen von (logarithmierter) Haushaltsverschuldung und (logarithmiertem) BGV anhand der österreichischen Mikrodaten mittels einer Kerndichteschätzung der Verschuldung und des BGV für alle verschuldeten Haushalte ermöglicht außerdem eine genauere Analyse des Zusammenhangs zwischen Haushaltsverschuldung und Geldvermögen (vgl. Abbildung I S. 42). Zudem können vergleichbare Kerndichteschätzungen aus Brown/Taylor (2005) für die USA, für Deutschland und für das Vereinigte Königreich herangezogen werden, ${ }^{8}$ um länderspezifische Unterschiede zu untersuchen. Wie sich zeigt, verfügen in Österreich nahezu alle verschuldeten Haushalte auch über ein positives BGV, beide Verteilungen weisen ein hohes Maß an Übereinstimmung auf. Demgegenüber verfügt in den USA ein nicht unbeträchtlicher Anteil der verschuldeten Haushalte über kein oder sehr geringes Geldvermögen, die Verteilung der Verschuldung liegt für die USA deutlich rechts der Verteilung des Geldvermögens. Wie Brown/Taylor (2005) feststellen, übersteigen für einen nicht unbeträchtlichen Teil der Haushalte im Vereinigten Königreich und in den USA die Schulden das Geldvermögen. ${ }^{9}$ Verschuldete Haushalte in den USA und dem Vereinigten Königreich weisen damit zwar eine effizientere Portfolioallokation auf, gleichzeitig sind sie damit aber auch einer stärkeren Belastung des Geldvermögens durch Verschuldung ausgesetzt, was höhere Risiken für die Finanzmarktstabilität impliziert. Die Situation in Deutschland liegt, mit einigen akzentuierten Abweichungen, näher an der österreichischen als an der in den USA und im Vereinigten Königreich.

Um für mögliche Zusammenhänge anderer Variablen mit dem Schuldenstand zu kontrollieren, die hinter der positiven Korrelation zwischen Schulden und Geldvermögen stehen könnten, ist eine umfassende ökonometrische Analyse notwendig. Nur zur Illustration wird an dieser Stelle ein Hinweis auf erste, sehr vorläufige Schätzungen aus einem umfangreicheren Projekt gegeben, dessen Ergebnisse 2008 vorliegen sollen: Der Gesamtschuldenstand, der Wohn- und/oder Konsumkredite beinhaltet, bezieht sich für Österreich auf 99I Haushalte

8 Die Schätzungen von Brown / Taylor (2005) beruhen aufDaten der Panel Study of Income Dynamics (USA), des British Household Panel Survey (Vereinigtes Königreich) und des German Socio-Economic Panel (Deutschland), die jeweils, wie die verwendeten österreichischen Daten, auch Grundlage für die LWS-Daten sind. Die Höhe der Schulden beziehungsweise des Geldvermögens können zwischen den Ländern allerdings nicht verglichen werden, da die Daten in verschiedenen Zeiträumen und in unterschiedlichen Währungseinheiten erhoben wurden.

9 Dieses Ergebnis gilt selbst unter Miteinbeziehung des Immobilienvermögens, also in Bezug auf das Gesamtvermögen der Haushalte. 


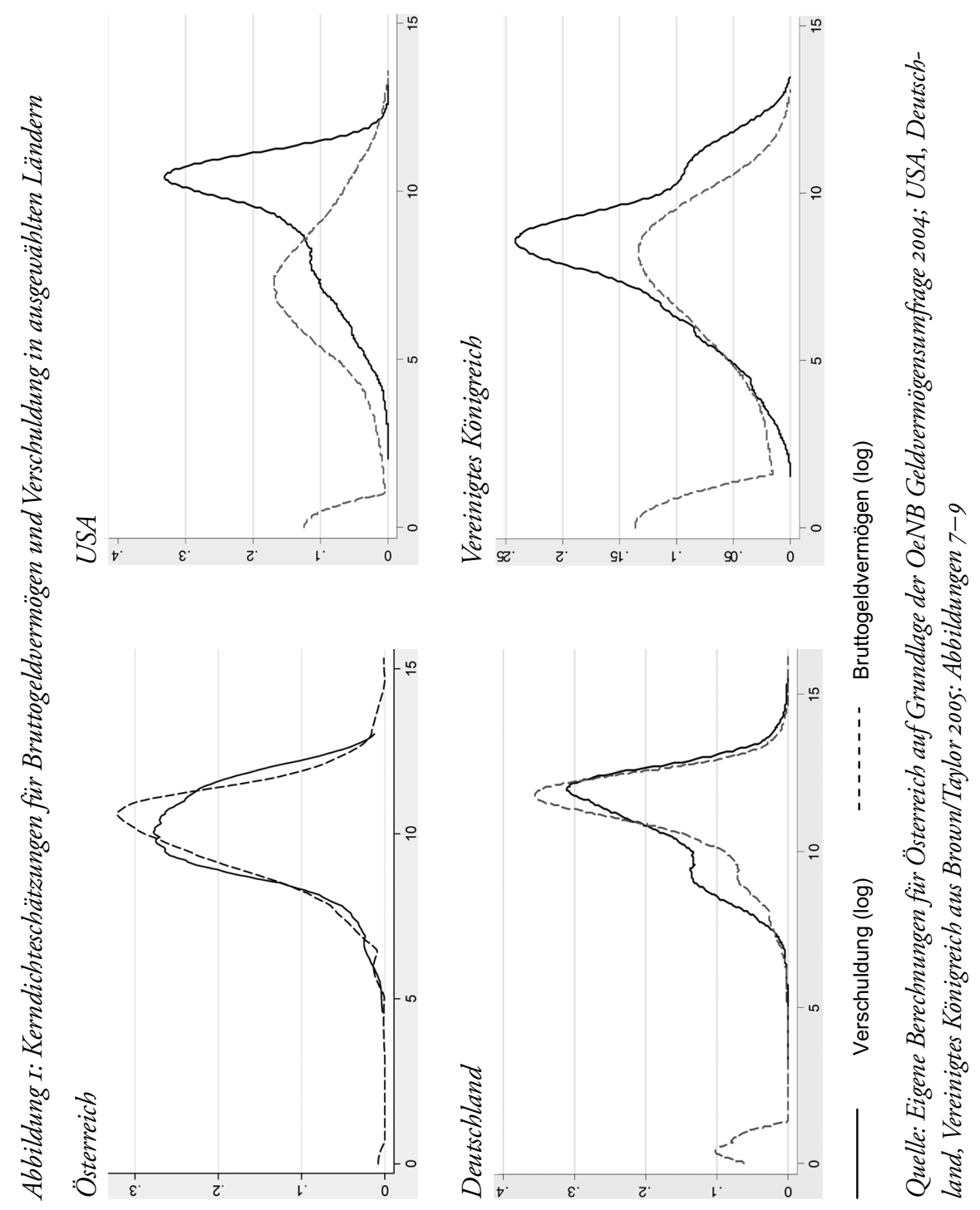


der Stichprobe, 1.565 Haushalte weisen keine Verschuldung auf. Das Einkommen liegt nur als kategoriale Variable vor, die die Haushalte nach Einkommensklassen ordnet. Die zentralen Variablen für den Zusammenhang zwischen Haushaltsverschuldung und Einkommen bzw. Geldvermögen sind das logarithmierte Bruttogeldvermögen und ein Vektor dreier Dummy-Variablen für Einkommensklassen. Als sozioökonomische Charakteristika werden zusätzlich das Alter des Hausvorstandes, die Anzahl der im Haushalt lebenden Personen und Dummy-Variablen für die Bildung des Haushaltsvorstandes, Unternehmerhaushalte ${ }^{\mathrm{io}}$ und Haushalte mit Erbschaften verwendet.

Wir schätzen zur Vermeidung eines möglichen Sample Selection Bias bei OLS-Schätzern zusätzlich ein Heckman Selection Model (vgl. Heckman 1979 und Vella 1998), bestehend aus einer Selektionsgleichung ${ }^{\text {II }}$, die die Entscheidung zur Verschuldung abbildet, und einer Regressionsgleichung, die die Höhe der Verschuldung erklärt. Wie erste Ergebnisse aller geschätzten Modellvarianten für Österreich zeigen, wird die Hypothese eines negativen Zusammenhanges zwischen Geldvermögen und Verschuldung für jene Haushalte, die Schulden halten, auch dann abgelehnt, wenn für eine Reihe relevanter anderer Variablen kontrolliert wird. Das steht im Einklang mit ähnlichen Ergebnissen zu einem positiven Zusammenhang zwischen Nettovermögen und (gewünschter) Verschuldung von Magri (2002) und Cox/Japelli (1993) für Italien beziehungsweise die USA.

\section{Schlussfolgerungen}

Der Zusammenhang zwischen Haushaltsverschuldung, Einkommen und Geldvermögen ist weit komplexer, als üblicherweise diskutiert und dargestellt. Allerdings ermöglichen nur - selten verfügbare-Mikrodaten eine ausreichend detaillierte Analyse der Thematik. Wie sich anhand dieser Daten für Österreich zeigt, werden Wohnkredite häufiger von Haushalten mit höherem Geldvermögen und höherem Einkommen gehalten, Konsumkredite hingegen häufiger von Haushalten mit niedrigerem Geldvermögen, aber ebenfalls höherem Einkommen. Wohnkredite dominieren in Verbreitung und Höhe die Verschuldungssituation des Haushalts. Auf die Wahrscheinlichkeit von Kreditverschuldung wirkt sich steigendes Geldvermögen - wenn für andere Variablen wie Einkommen, Bildung, Alter, Anzahl der Personen im Haushalt kontrolliert wird - erwartungsgemäß negativ aus. Innerhalb der Gruppe der verschuldeten Haushalte kann ein negativer Zusammenhang des Geldvermögens mit der Höhe der Schulden aber nicht nachgewiesen werden. Je mehr Geldvermögen verschuldete Haushalte halten, desto höher ist auch ihr Schuldenstand. Dies kann als suboptimale Allokation innerhalb der Haushaltsportfolios interpretiert werden, insbesondere

Io Haushalte, in denen mindestens eine Person an einem Unternehmen beteiligt ist.

II In der Selektionsgleichung werden zusätzlich die Dummy-Variablen für den Wohnsitz in Wien und verheiratet/nicht verheiratet (in Bezug auf den Haushaltsvorstand) verwendet. Dabei bestätigt sich, dass die Wahrscheinlichkeit Schulden zu halten mit steigendem Geldvermögen sinkt. Es ist uns zudem nicht möglich, Angebots- und Nachfrageseite zu trennen, was die Interpretation insbesondere der Selektionsgleichung schwierig macht, da die meisten der verwendeten Variablen sowohl für die Angebots- als auch die Nachfrageseite eine Rolle spielen können. 
im Bereich höherer Vermögen. Der Schuldenstand verschuldeter Haushalte wird zudem von Einkommen und Bildung positiv beeinflusst. Generell wird deutlich, dass sich der Schuldenstand eines Haushaltes nicht als "Belastungsindikator « eignet, wie in ähnlicher Weise auch die in der Literatur verbreitete Verwendung der Relation zwischen Verschuldung und Einkommen. Verschuldung sollte vielmehr immer gemeinsam mit dem Gesamtvermögen des Haushalts und seinem relativ liquiden Geldvermögen analysiert werden. Vorläufige empirische Evidenz deutet darauf hin, dass Haushalte mit niedrigeren Schuldenständen, die vorrangig Konsumkredite halten, tendenziell am stärksten durch Verschuldung belastet sind. Gleichzeitig ergibt sich für Österreich aus der steigenden Verschuldung privater Haushalte kaum ein erhöhtes Risiko für die Finanzmarktstabilität, da - im deutlichen Unterschied zu den USA und zum Vereinigten Königreich-die Haushalte mit höherer Verschuldung auch über entsprechendes Geldvermögen verfügen.

\section{Literatur}

Aizcorbe, Ana M. / Kennickell , Arthur B. / Moore, Kevin B. (2003): Recent Changes in U.S. Family Finances: Evidence From the 1998 and 200I Survey of Consumer Finances, in: Federal Reserve Bulletin, Nr. I, S. I-32

Barnes, Sebastian/Young, Garry (2003): The Rise in US Household Debt: Assessing Its Causes and Sustainability, Bank of England Working Paper, Nr. 206

Beer, Christian / Mooslechner, Peter / Schürz, Martin / Wagner, Karin (2006): Das Geldvermögen privater Haushalte in Österreich: Eine Analyse auf Basis von Mikrodaten, in: Geldpolitik und Wirtschaft, Nr. 2, S. IOI-II4

Beer, Christian/Schürz, Martin (2007): Charakteristika der Verschuldung der privaten Haushalte in Österreich: Ist die Verschuldung ein Risiko für die Finanzmarktstabilität?, in: Geldpolitik und Wirtschaft, Nr. 2, S. 62-83

Brown, Sarah/Taylor, Karl (2005): Household Debt and Financial Assets: Evidence From Great Britain, Germany and the United States, University of Leicester Working Paper, Nr. 05/5

Browning, Martin / Lusardi, Annamaria (1996): Household Saving: Micro Theories and Micro Facts, in: Journal of Economic Literature, Jg. 3, Nr. 4, S. $1797-1855$

Cox, Donald / Japelli, Tullio (1993): The Effect of Borrowing Constraints on Consumer Liabilities, in: Journal of Money, Credit, and Banking, Jg. 25, Nr. 2, S. 197-213

Cox, Pru/Whitley, John/Brierley, Peter (2002): Financial Pressure in the UK Household Sector: Evidence From the British Panel Survey, in: Bank of England Quarterly Bulletin, S. $4 \mathrm{IO}-4 \mathrm{I} 9$

Crook, Jonathan (2003): The Demand and Supply for Household Debt: A Cross Country Comparison, Credit Research Centre, University of Edinburgh Working Paper Series, Nr. O3/OI

Girouard, Nathalie/Kennedy, Mike/André, Christophe (2006): Has the Rise in Debt Made Households More Vulnerable?, OECD Economics Department Working Papers, Nr. 535 
Heckman, James J. (1979): Sample Selection Bias as a Specification Error, in: Econometrica, Jg. 47 , Nr. I, S. I53-I6I

Laibson, David (1997): Golden Eggs and Hyperbolic Discounting, in: Quarterly Journal of Economics, Jg. II2, Nr. 2, S. $443-477$

Laibson, David/Repetto, Andrea/Tobacman, Jeremy (2000): A Debt Puzzle, National Bureau of Economics Research Working Paper, Nr. 7879

Magri, Silvia (2002): Italian Households' Debt: Determinants of Demand and Supply, Banca d'Italia, Temi di discussione del Servizio Studi, Nr. 454

Modigliani, Franco / Brumberg, Richard (1954): Utility Analysis and the Consumption Function: An Interpretation of the Cross-Section Data, in: Post-Keynesian Economics, New Brunswick: Rutgers University Press, S. $338-436$

Thaler, Richard H. (1990): Anomalies: Saving, Fungibility, and Mental Accounts, in: The Journal of Economic Perspectives, Jg. 4, Nr. I, S. 193-205

Vella, Francis (1998): Estimating Models with Sample Selection Bias: A Survey, in: The Journal of Human Resources, Jg. 33, Nr. I, S. 127-169

\section{Does Globalization Make the World More Equitable? Kurt Bayer*}

\section{Trends in Globalization}

Globalization is not a new phenomenon. A first phase occurred during the colonization of Africa, Asia and Latin America when raw materials for Europe were extracted, slave labor shipped across the world and markets developed as outlets for European commodities. This critical phase can be dated from the I850s to World War I. During that time the share of exports in world GDP doubled to eight percent, 60 million Europeans emigrated to America, similar numbers from China and India to other South Asian and African countries. Migration flows reached ten percent of world population, trade relations flourished. This trend reversed after World War I: by the I950s, the share of exports in world GDP had fallen back to its I870 level of four percent, per capita growth was lower by one third, partly as a result of the wars, partly as a result of the breakdown of trade and investment relations due to protectionist policies and a dramatic fall in world demand in the course of the Great Depression.

* European Bank for Reconstruction and Development, London.

(C) INTERVENTION 5 (I), 2008, 45-54 